\title{
Optimizing an electrochemical deposition process by use of design of computer experiments
}

\author{
D. Rabiot ${ }^{1}$, J.-P. Caire ${ }^{1, *}$, B. Nguyen ${ }^{1}$, E. Chainet ${ }^{1}$ and F. Gerard ${ }^{2}$ \\ ${ }^{1}$ LEPMI, ENSEEG, UMR 5631 INPG-CNRS, Domaine Universitaire, 1130 rue de la piscine, BP. 75, \\ 38402 Saint Martin d'Hères Cedex, France \\ ${ }^{2}$ Alcatel Alsthom Recherche, Route de Nozay, 91460 Marcoussis, France
}

\begin{abstract}
A response surface methodology only based in this case on computer experiments was used in conjunction with the desirability concept to optimize a priori an electrodeposition process designed for production of gold-tin alloy bumps on a 4 inches wafer. The efficiency of the method was used to achieve at a cheap cost with a small number of computer experiments the technical specifications required in term of metal deposit thickness uniformity.
\end{abstract}

Key words. Electrodeposition process - computer experiments - design of experiments - response surface methodology desirability functions.

\section{Introduction}

Process optimization is essential for cheap and rapid process technology development. Experimental designs have found widespread applications in the physical sciences. Since a computer simulation experiment is indeed an experiment, the response surface methodology can be used for numerical experiments. Hunter JS [1] was probably the first author in 1970 to point out the advantages and problems related to computer aided simulation. The concept of desirability functions used in conjunction with experimental designs was described in 1965 by Harrington EC [2]. Coupling both concepts of numerical design of experiments and desirability functions to optimize a physical system was surprisingly used in very few papers essentially devoted to semiconductor technology [3-11].

Efficient numerical simulation tools have been developed recently to compute potential and current distributions in an electrochemical cell. Improvement of the cell design can be achieved in a convenient way by varying, one variable at a time, the geometrical parameters of the cell, but such a method may lead to numerous time consuming trials and cannot assure that the optimum design is actually obtained. The so-called Response Surface Methodology (RSM) [12] based on design of experiments [13] both reduces drastically the number of computer trials needed to optimize the process and gives statistical inference on the optimum.

This paper presents the optimizing process of a cell geometry designed to obtain an uniform electrodeposition on a 4 inches wafer. A five parameters central composite in a cube design [13] was used for the RSM. This design made by use of the personal computer software Echip6 ${ }^{\circledR}$ [14] led to 27 computer experiments requiring for each simulation one hour of computing time using a IBM Risc 6000 workstation with the Flux-Expert ${ }^{\circledR}$ commercial code [15]. Several different responses of interest were analyzed with Echip6 ${ }^{\circledR}$ [14] and were combined by use of the desirability concept [2] to obtain pertinent criteria to be optimized. The RSM methodology can show which variables are influent and how they interact, and leads to the best satisfying design.

* Correspondence and reprints.

Received January 13, 1998; revised May 13, 1998; accepted May 20, 1998. 


\section{Original articles}

\section{Problem and method}

The electrodeposition process studied here allows the deposition of successive thin gold and tin layers on the support, an alloy being then obtained by fusion for the realization of small gold-tin bumps. Though this electrochemical process is reliable and precise, thickness non-uniformity occurs during metal deposition and leads to local variation of alloy composition related to eutectic temperature problems during the fusion process.

This lack of uniformity is commonly encountered for electrochemical cells, especially in the semiconductor industry and has been very precisely described by Dukovic [16]. It is linked to a concentration of current density lines at the wafer edges. This is the well-known edge effect, leading to a thickness deposit larger in the peripheral part of the wafer than in its center (Fig. 1).

To limit these edge effects, classical solutions such as utilization of so-called insulating walls, current thieves or auxiliary electrodes have been proposed in the past years [1718]. The two last solutions are not applicable in this case due to the financial costs associated with gold deposition.

For the same reasons, the experimental study of numerous different geometrical configurations for such a process is a long and expensive task. In order to optimize the cell design, use of design of experiments coupled with desirability functions decreases drastically the number of trials and reduces the effective costs. Then numerical experimentation appears as a more preferable mean to screen numerous trial configurations.

This study intends to optimize by numerical calculation the geometrical configuration of the cell in order to minimize edge effects. The first step of the study has consisted in obtaining a numerical model to correctly represent the reality. Then the existent cell was modeled with the help of Flux-Expert ${ }^{\circledR}$, a commercial software based on the finite element method [15]. This code solves the Laplace equation and computes potential and current distributions in the cell (Fig. 2). The profile of metal thickness is then deduced from current distribution by use of the Faraday law assuming a $100 \%$ current efficiency in the case of gold deposition. Due to the axial symmetry of the cell, and to lower the computing time, only half a cell is modeled here (Fig. 3).

The validation of the numerical model was made for the existing cell. Some geometrical interesting configurations with and without insulating mask placed in front of the cathode were evaluated with the help of a Plackett-Burman [13] screening design not presented here.

This screening design showed that the presence of an insulating mask gave better uniformity results. It also allowed to retain at last the five geometrical factors appearing in figure 3 namely: the anode diameter (variable named "dianode"), the internal diameter of the mask ("diamasque"), the thickness of the mask ("epesmasc"), the distance between the mask and the cathode ("mascatod"), the distance between the anode and the cathode ("entrode"). The experimental domain of variation of each parameter was carefully chosen to fulfill the physical constraints associated to a realistic cell.

A five variables Box-Wilson central composite in a cube design was used to optimize the geometry [12-13]. Such a

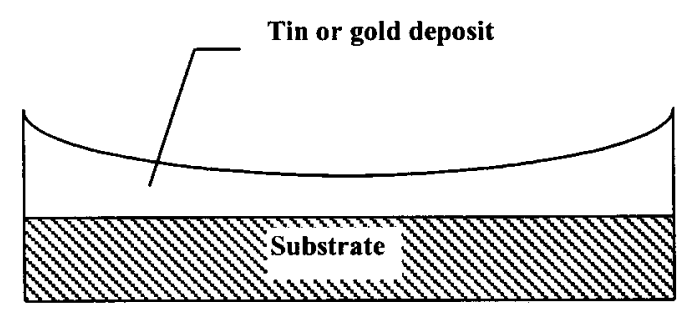

Fig. 1. Metal deposit profile.

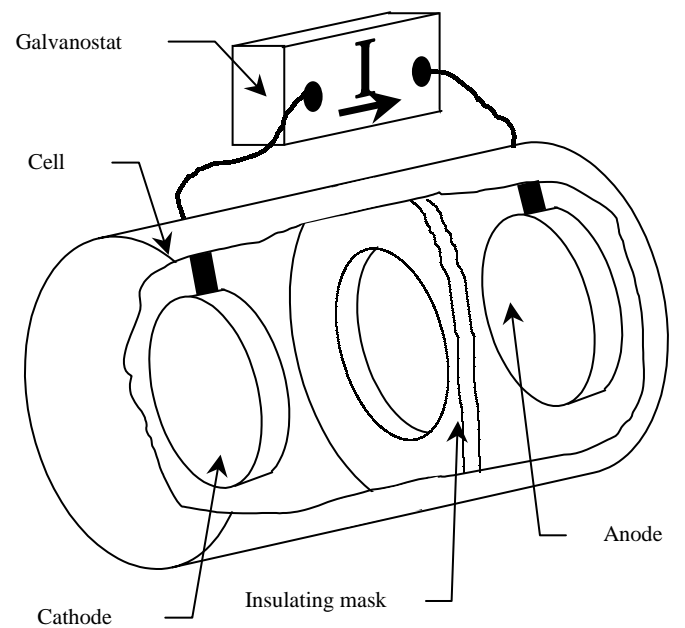

Fig. 2. Schematic 3D view of the cell.

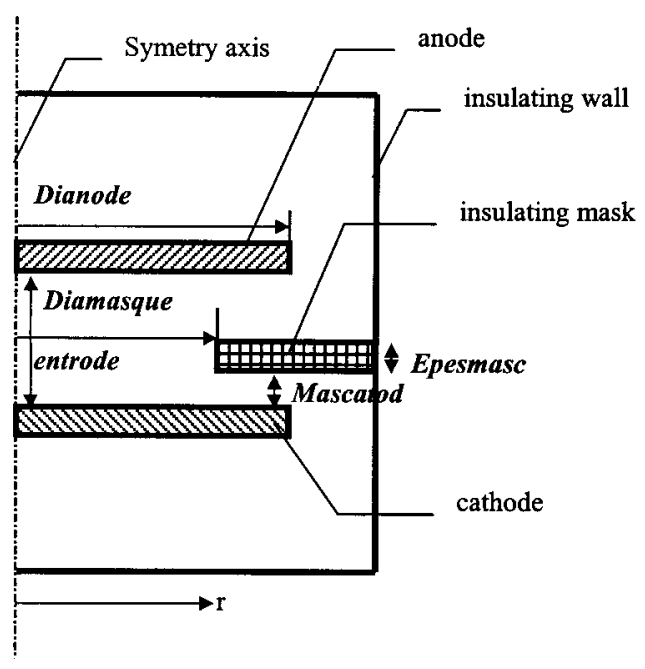

Fig. 3. Schematic presentation of the different parameters of the studied cell.

design of experiments was chosen for its favorable properties:

- it provides the quadratic polynomial models indispensable for the search of optima with the response surface methodology [12], 


\section{Original articles}

- the use of only three level variables facilitates the implementation of numerical simulation input files,

- it requires only 27 calculations including the center point.

The last point is a very important one since each individual run lasts one hour on a IBM Risc 6000 workstation.

The responses of interest were:

- the radial cathode current density distribution $J_{(r)}$ along a radius of the cathode (it is directly linked to the thickness of the metal deposit by the Faraday law),

- the global cell voltage $U$,

- the global uniformity $\sigma$ of metal deposit represented by the thickness standard deviation calculated on the whole wafer,

- a useful wafer radius $x$, expressed in $\mathrm{mm}$, and defined as the central part of the wafer where the $\left(J_{(r)}-J_{(r=0)}\right) / J_{(r=0)}$ ratio is smaller than $10 \%$, this specification being convenient for the process.

\section{Numerical results}

Both creation and treatment of the design of numerical experiments were made with the help of Echip6 ${ }^{\circledR}$. For each simulation, the boundary conditions were the following : a total current intensity $I$ was imposed on the anode and a null arbitrary potential was used as a Dirichlet condition on the cathode. The cathode current density $J_{(r)}$ was obtained from the resolution of Laplace equation. The responses $J_{(r)}, U, \sigma$ and $x$ were then calculated for each run and introduced in the Echip6 ${ }^{\circledR}$ code.

A statistical study of residuals was carefully made in order to assure that the empirical quadratic models associated to each response were pertinent. Then an optimal compromise was obtained for both responses $\sigma$ and $x$ by use of the combined response optimization tool defined in Echip6 ${ }^{\circledR}$. This tool allows the user to weight freely the desirability functions associated to each individual response. For example, a larger weight was attributed here to the response $x$ which has a greater importance from an industrial point of view. The optimal compromise is then obtained from the global desirability function to be minimized. The optimum found by Echip6 ${ }^{\circledR}$ was pointed out on contour plots in figure 4 .

As seen in figure 4, the optimum appears at the border of the experimental domain. A better result could theoretically be obtained outside the design, but this part of space is not really available for industrial and physical constraints.

A new computer experiment using Flux-Expert ${ }^{\circledR}$, was made with the optimal set of parameters to verify the theoretical optimal geometry and a very good agreement was found with the model prediction. The table I shows the results obtained for the optimal set of parameters.

\section{Conclusions}

The response surface methodology using computer experiments coupled with desirability concept has allowed to obtain in a very restrained number of both rapid and cheap numerical experiments a satisfying compromise in term of

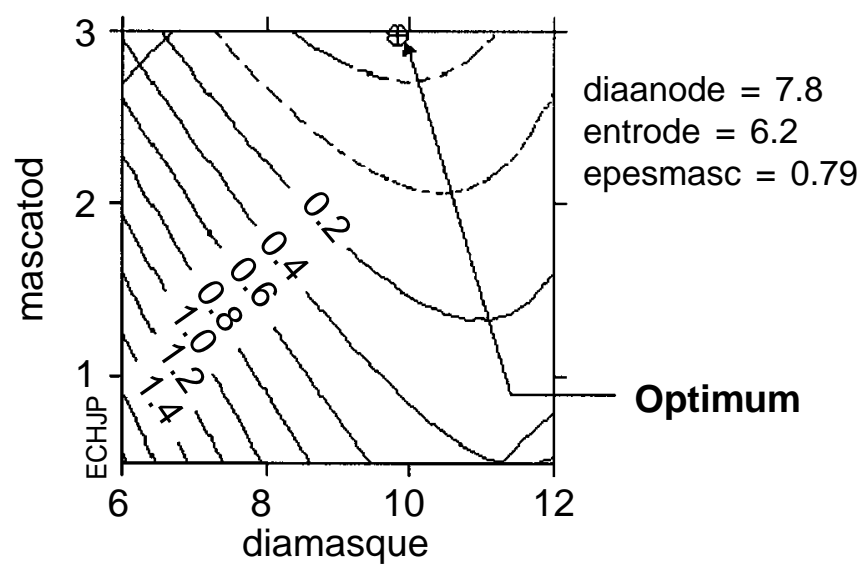

Fig. 4. Uniformity contour plots and the optimal geometry (the parameters are given in $\mathrm{cm}$ ).

Table I. Least optimal numerical results.

\begin{tabular}{lc}
\hline Responses & Value \\
\hline$\sigma / \mathrm{mm}$ & 0.051 \\
$x / \mathrm{mm}$ & 46.19 (for a 50 mm radius) \\
Mean deposit thickness/um & 2 \\
$U / V$ & 0.19 \\
\hline
\end{tabular}

electrode current density and deposit uniformity. The primary technical objectives are obtained in 28 CPU hours and moreover, thanks to the response surface methodology, the limitations of the possible cell improvements have been clearly identified.

\section{References}

1. Hunter, J. S.; Taylor, N. H. Manag. Sci. 1970, 16, 422-434.

2. Harrington, E. C. Indus. Qual. Cont. 1965, 4, 494-496.

3. Cartuyvels, R.; Booth, R.; Dupas, L.; De Meyer, K. Microelectr. Eng. 1992, 19, 507-510.

4. Cechetti, M.; Lissoni, M.; Lombardi, C.; Marmiroli, A. Microelectr. Eng. 1992, 19, 511-516.

5. Otten, J. A. M.; Otten, G. M. J.; Klaassen, F. M. Improvement of LDD MOSFET's characteristics using an inverse modeling technique for device and process optimization.

6. Alvarez, A. R.; Abdi, B. L.; Young, D. L.; Weed, H. D.; Herald, E. R. IEEE Trans. Comp.-Aided Design 1988, 7, 272288.

7. Low, K. K.; Director, S. W. IEEE Trans. Comp.-Aided Design 1989, 8, 1299-1313.

8. Biles, W. E. Design of simulation experiments, Proceeding of the 1984 Winter simulation conference, Sheppard, S.; Pooch, U.; Pedgen, D. Eds.

9. Hanrahan, J. J.; Baltus, T. A. IEEE Trans. Indus. Appl. 1992, 28, 293-296.

10. Kump, M. R. K.; Mylrole, S. W. Use of process simulators to assist in the design of processes for manufacturability, IEEE/SEMI Advance Semiconductor Manufacturing Conference, 1990; pp 15-21.

11. Bagghi, T. P.; Kumar, M. R. J. Electr. Manufact. 1993, 3, 3138. 


\section{Original articles}

12. Box, A. R.; G. E.P.; Draper, N. Empirical model-building and response surfaces. John Wiley, NY, 1987.

13. Box, G. E. P.; Hunter, W. G.; Hunter, J. S. Statistics for experimenters, John Wiley, NY, 1978.

14. Echip Inc., Release 6, 724 Yorklyn Road, Hockessin, DE 19707-8703, USA.

15. Flux-Expert, Simulog, Miniparc Bâtiment 1, 60 rue Lavoisiser, 38330 Montbonnot, France.
16. Dukovic, J. O. Adv. Electrochem. Eng. VCH, 1990, 3, 117-161.

17. Mehdizadeh, S.; Dukovic, J.; Andricacos, P. C.; Romankiv, L. T.; Cheh, H. Y. J. Electrochem Soc. 1990, 137, 110-117.

18. Choi, Y.- S.; Kang, T. J. Electrochem. Soc. 1996, 143, 480485. 